\title{
Semiconductor Gas Sensors to Analyze Fecal Exhalation as a Method for Colorectal Cancer Screening
}

\author{
G. Zonta 1,2, , M. Astolfi 1,2, A. Gaiardo ${ }^{1,2,4}$, S. Gherardi ${ }^{2}$, A. Giberti ${ }^{2,3}$, V. Guidi ${ }^{1}$, N. Landini ${ }^{1,2}$ \\ and C. Malagù 1,2 \\ 1 University of Ferrara, Via Savonarola 9, 44121 Ferrara, Italy; michele.astolfi@unife.it (M.A.); \\ andrea.gaiardo@unife.it (A.G.); guidi@fe.infn.it (V.G.); nicolo.landini@unife.it (N.L.); \\ malagu@fe.infn.it (C.M.) \\ 2 SCENT S.r.l., Via Quadrifoglio 11, 44124 Ferrara, Italy; gherardi@fe.infn.it (S.G.); giberti@fe.infn.it (A.G.) \\ 3 MIST E-R s.c.r.l., Via P. Gobetti 101, 40129 Bologna, Italy \\ 4 MNF - Micro Nano Facility, Bruno Kessler Foundation, Via Sommarive 18, 38123 Trento, Italy \\ * Correspondence: giulia.zonta@unife.it; Tel.: +39-0532-974-286 \\ + Presented at the 8th GOSPEL Workshop. Gas Sensors Based on Semiconducting Metal Oxides: Basic \\ Understanding \& Application Fields, Ferrara, Italy, 20-21 June 2019.
}

Published: 19 June 2019

\section{Introduction}

Colorectal cancer (CRC) preventive screening is fundamental to identify tumors before their possible degeneration. The current screening method employed on population aging between 50-69 years by Italian National Health Service is fecal occult blood test (FOBT). This test leads to a lot of false positives (more than $60 \%$ ) and to non-operative colonoscopies.

Our patented device, named SCENT A1 [1] and described in our publications [2-4], is capable to identify CRC with an economic, in-vitro, non-invasive technique. The device is composed of a set of five chemoresistive MOX sensors that, by smelling the odor of fecal samples, can distinguish between populations of healthy and CRC-affected patients. The odor of fecal exhalations is strongly different if the tumor is present, also depending on its dimensions and degeneration stage, due to the presence of specific tumor biomarkers produced by peroxidation of the cell membrane or by metabolic alterations. Here the results obtained so far in the clinical validation Protocol, started in May 2016, that involves S. Anna Hospital of Ferrara, UNIFE, Ospedale del Delta of Lagosanto, AUSL of Ferrara and the startup SCENT S.r.l.

\section{Experimental Section}

All screening users in Ferrara who resulted positive to FOBT can participate in the project bringing an additional sample of frozen feces to be analyzed with SCENT A1. Up to now, over 500 fecal samples have already been measured with the device, already resulted positive for FOBT, 100 of which were already compared with colonoscopy. The technique employed for data analysis is support vector machine (SVM) by dividing the samples into three categories (low-risk adenomas, LR, healthy subjects, NEG, and high-risk adenomas plus carcinomas), see Figure 1. Here this method correctly classified the $90 \%$ of NEG, the $100 \%$ of CRC and the $57 \%$ of LOW. A new approach, that employs the information of only two sensors, to simplify the system and improve the algorithm efficiency, is the k-fold cross validation test. This method ensures an immediate double-blind check. It emerges that, by performing SCENT A1 test with the data compared so far, the percentage of colonoscopies saved is $79 \%$. If the results over a sufficiently large statistics will be confirmed, the goal 
would be to combine FOBT with SCENT A1 test in the countries where FOBT is employed as a screening on population.

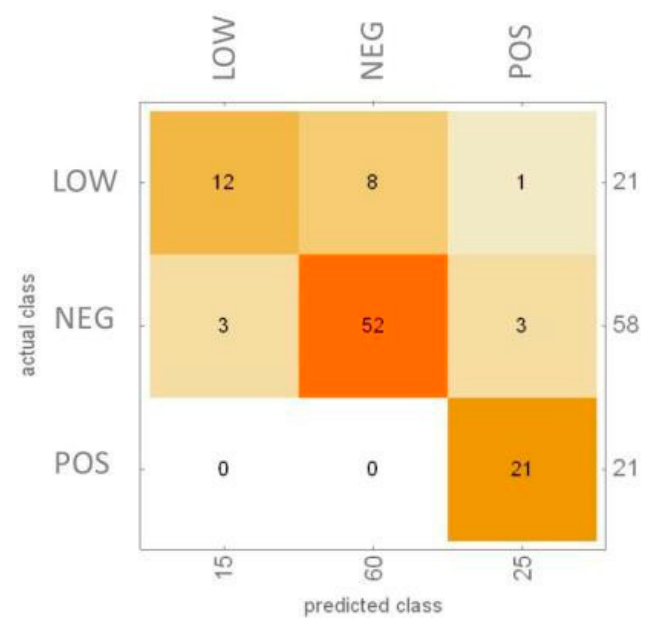

Figure 1. Confusion matrix with 100 samples.

Funding: This research was supported by LILT (Lega Italiana Lotta Tumori) section of Ferrara for the Design Work Plan Call for Health Research 2016 "Programma 5 per mille anno 2014".

Acknowledgments: The authors would like to thank SCENT S.r.l. for the operational contribution in the research.

Conflicts of Interest: The authors declare no conflict of interest.

\section{References}

1. SCENT A1. Italian patent number: RM20144000595, European extension: 3210013; patent of SCENT S.r.l.

2. Landini, N.; Zonta, G.; Malagù, C. Detection of Tumor Markers on Feces with Nanostructured Sensors; Scholars' Press; Saarbrucken, Germany, 2015; ISBN-13: 978-3-639-76538-0.

3. Zonta, G.; Anania, G.; Fabbri, B.; Gaiardo, A.; Gherardi, S.; Giberti, A.; Landini, N.; Malagù, C.; Scagliarini, L.; Guidi, V. Preventive screening of colorectal cancer with a device based on chemoresistive sensors. Sens. Actuators B 2016, 238, 1098-1110.

4. Zonta, G.; Anania, G.; Feo, C.; Gaiardo, A.; Gherardi, S.; Giberti, A.; Guidi, V.; Landini, N.; Palmonari, C.; Ricci, L.; de Togni, A.; Malagù, C. Use of gas sensors and FOBT for the early detection of colorectal cancer. Sens. Actuators B 2018, 262, 884-891.

() 2019 by the authors. Licensee MDPI, Basel, Switzerland. This article is an open access article distributed under the terms and conditions of the Creative Commons Attribution (CC BY) license (http://creativecommons.org/licenses/by/4.0/). 